\title{
Management of treatment resistant schizophrenia
}

\section{S Jones ${ }^{1}$, DJ Castle ${ }^{2}$}

${ }^{1}$ Royal Melbourne Hospital/ North-West Area Mental Health Service, Melbourne, Australia

${ }^{2}$ Mental Health Research Institute and University of Melbourne, Australia

\begin{abstract}
Whilst gains have been made in recent years in the pharmacological treatment of schizophrenia, a number of patients still have residual symptoms and disabilities, or simply do not show response to antipsychotic medications. For such 'treatment resistant' patients, there is little by way of randomised controlled data to support any particular type of further intervention, but combinations of agents (combined antipsychotics, augmentation with mood stabilisers, antidepressants, and other agents)can show benefit in certain patients in certain domains of symptomatology and psychosocial functioning. Certain psychological and psychosocial treatment strategies can also be of benefit in this regard. This article selectively reviews the literature treatment resistance in schizophrenia, and emphasises the importance of an holistic approach to individual patients.
\end{abstract}

Keywords: Schizophrenia; Treatment resistance; Antipsychotics; Augmentation; Psychosocial treatments

Received: 26.04 .05

Accepted: 31.05 .05

\section{Introduction}

The concept of treatment resistance in schizophrenia has evolved considerably in recent years. The emphasis of earlier studies was on positive psychotic symptoms and their response to antipsychotic medication. Thus, a person who failed to have an adequate response (resolution of psychotic symptoms) to two different anti-psychotics was considered to be 'treatment resistant'. While this is still used in many current guidelines as a rationale for commencing clozapine, the overall concept of treatment resistance has broadened considerably. Consideration is now given to a number of domains other than positive symptoms, including negative symptoms, cognitive deficits, excitement or aggression, comorbidity such as depressive and anxiety symptoms, and functional level or disability. ${ }^{1}$

This shift in emphasis reflects our changing understanding of the illness, the increased relevance of broader life domains since de-institutionalisation ${ }^{2}$, expansion of treatment beyond pharmacotherapy to include psychological and occupational

\section{Correspondence:}

Prof DJ Castle

155 Oak Street, Parkville, Victoria, 3052, Australia

email: dcastle@mhri.edu.au interventions, and a greater appreciation of patients' subjective experience of the recovery process. This is in parallel with the emergence of the 'consumer movement'. Thus, concepts such as realistic hope, the desire to achieve autonomy/self-determination and quality of life are increasingly the currency of comprehensive treatment outcomes in schizophrenia. ${ }^{3}$ Indeed, some authors suggest that "treatment resistance" should be replaced with a term such as "incomplete recovery" given the former's implication that the patient is resisting treatment rather than the illness being resistant and the fact that the latter better promotes an identification of barriers to recovery. ${ }^{2}$

The field also needs to consider the issue of what is a reasonable expectation of 'outcome' and consideration of 'recovery vs cure' in areas such as symptoms, service utilisation, quality of life, work and relationships. Peuskens ${ }^{4}$ suggests that a person's response to treatment cannot be viewed as a dichotomy of 'response or no-response' and should be considered as a continuum.

Clearly such changes in conceptualisation and the expansion of the concept of treatment resistance makes it the "rule rather than the exception". 5 This is consistent with outcome studies suggesting that only $20-30 \%$ of people with schizophrenia have complete remission of symptoms and 'good outcome'. ${ }^{6}$ Moreover, it reflects the heavy burden of disease that is present worldwide, as well as more controversial propositions that even with optimal treatment 
only about $20 \%$ of the burden of disease associated with schizophrenia can be alleviated. ${ }^{7}$ Whatever the figures may be, it is clear that treatment resistance has major health, economic and social consequences. Also, common sequelae such as homelessness, itinerancy and incarceration lead to further difficulties with inadequate treatment access and response. ${ }^{1}$

There are several well recognised factors associated with poorer outcome in people with schizophrenia that may be relevant when considering treatment resistance. Some of these parameters (Table I) ${ }^{1}$ are not readily amenable to change but others may be reversed or at least ameliorated (Table II) and highlight the importance of a comprehensive assessment and management plan.

\section{Table I: Factors Associated With Poor Outcome in Schizophrenia}

Male sex

History of obstetric complications

Early or insidious onset

Lack of precipitating factors

Poor pre-morbid functioning

Increased number of episodes of psychosis

Long duration of untreated psychosis

Severe negative symptoms

Absence of affective symptoms

Neurological soft signs

Significant neuro-cognitive deficits

Structural brain abnormalities

\section{Table II: Potentially remediable factors in patients with apparent} treatment resistance

Suboptimal treatment

- Psychosis

- Comorbid symptoms (eg depression, anxiety)

Poor treatment adherence

Side effects of medication

Substance abuse

High family expressed emotion (EE)

Poor therapeutic alliance

Vocational impairment

Itinerancy and homelessness

We must appreciate that there is seldom a "quick fix" and that people with schizophrenia require interventions that are "sustained, comprehensive, co-ordinated, collaborative and consumer oriented". ${ }^{3}$ In order to plan such interventions, there clearly needs to be a detailed assessment of issues needing to be addressed. Such an assessment should take the concept of treatment resistance well beyond positive psychotic symptoms and simply response to anti-psychotic medication. It leads to the consideration of a range of other interventions that have been shown to be of potential utility in addressing some of the broader disabilities associated with schizophrenia.

\section{Medication and Treatment Resistance}

The published literature regarding the efficacy and effectiveness of different medications for treatment resistant schizophrenia has several limitations. As discussed above, the definition of what constitutes a treatment resistant "case" is complex and variable. Whereas some studies concentrate on strict definitions of positive symptoms, others are less clear or may also include a broader range of treatment resistant domains. For example, criteria for inclusion in Kane's often cited study ${ }^{8}$ comparing clozapine and chlorpromazine included: i. positive symptoms; ii. severity of illness on Brief Psychiatric Rating Scale (BPRS) and Clinical Global Impression Scale; and iii. social and occupational functioning. Other studies have included measures of negative symptoms and depression (eg. PANSS and MADRS respectively). ${ }^{9}$

There is an additional problem in measurement of outcomes. For example, when examining the effects of clozapine and olanzapine on negative symptoms, there have been variable results. It has been suggested that this may reflect differences in baseline levels of extra-pyramidal sideeffects (EPSE) and that observed reductions in negative symptoms may reflect an improvement in EPSE rather than a direct amelioration of negative symptoms. ${ }^{10}$ Thus one needs to clearly define primary negative symptoms (due to the primary illness, schizophrenia) and secondary negative symptoms (due to other factors such as medication sideeffects). Furthermore, studies have often not adequately addressed the possibility of other confounding factors contributing to apparent treatment resistance. For example, not all studies comprehensively assess factors such as treatment adherence, substance abuse or family expressed emotion, and those that do often use un-validated methods to monitor these during the course of treatment.

A majority of published medication trials in treatment resistant schizophrenia have examined differences between second generation anti-psychotics (SGAs) or 'atypicals' (including clozapine, olanzapine, risperidone, quetiapine, amisulpride and aripiprazole) and first generation antipsychotics (FGA.s) or 'typicals'. In a review and meta-analysis, Chakos et $\mathrm{al}^{10}$ found 12 controlled, randomized trials (of which 11 were double blinded) examining medication interventions for treatment resistant schizophrenia. Of these, seven compared the efficacy of clozapine to FGAs and three others compared other SGAs to FGA.s. Only two studies compared atypical agents with each other, in both cases risperidone and clozapine. The meta-analysis revealed that there was minimal to moderate improvement on SGA.s compared to FGA.s but that subjects still experienced many residual symptoms and impairments. The authors concluded that there was "insufficient evidence to definitively evaluate SGAs other than clozapine in the 'treatment resistant population".".

\section{Evidence for Specific Medications}

Clozapine is the best studied antipsychotic medication for treatment resistant schizophrenia. Treatment guidelines recommend that clozapine should be offered to 'all patients who have not responded to an adequate trial of two or more other antipsychotic drugs, at least one of which should be a SGA... A reasonable trial period of at least 6-12 months should occur, after which time approximately one-third of patients should show at least moderate improvement.' ${ }^{11}$

Studies that underpin such recommendations consist predominantly of comparisons of clozapine with FGAs and have mostly been of relatively short duration. For example, the two widely cited studies of Kane et $\mathrm{ll}^{8}$ (vs chlorpromazine) and Brier et $\mathrm{al}^{9}$ (vs haloperidol) were of 6 weeks and 10 weeks respectively. Response rates of $30 \%$ vs $4 \%$ and $44 \%$ vs $6 \%$ respectively may thus be underestimates, with the possibility of enhanced efficacy should the patients have been followed up over longer periods. Indeed, many clinicians report 
ongoing improvement over months or years on clozapine. Of the studies included in the Chakos et al review, only two of the double-blinded trials went beyond 12 weeks. In the same review, results of five of the seven studies favoured clozapine (improvement in BPRS total score) but had a variable effect size $(0.14-0.8)$ which perhaps reflects the difficulties with studying this population group.

Only four studies ${ }^{10}$ specifically examined negative symptoms and according to the meta-analysis, there was no significant reduction in these symptoms with clozapine. The authors concluded that this result may have been due to insufficient statistical power. Comparison of other studies was complicated by difficulty differentiating between primary and secondary negative symptoms. ${ }^{13}$ In a population of adolescents with few extra-pyramidal side-effects, for example, there was evidence that clozapine can reduce primary negative symptoms. ${ }^{14}$ Other potential benefits of clozapine include a reduction in aggressive and suicidal behaviours. ${ }^{15}$

The evidence for the efficacy of SGAs other than clozapine in the treatment resistant population is much more mixed. Again, definitions of 'treatment resistance' vary and most studies are short term. For example, while risperidone (6mg) was significantly better tolerated with fewer extra-pyramidal side-effects than haloperidol (15mg) the improvement in symptoms was not sustained beyond four weeks. ${ }^{16}$ Results for olanzapine in treatment resistant patients are inconsistent, which may reflect differences in populations studied. For example, one study recruited long-term institutionalised patients ${ }^{17}$ whereas another included outpatients and less severely impaired individuals. ${ }^{9}$ Also, the outcome measures in these two studies differed and the high rate of haloperidol response in the latter study (36\% compared to $45 \%$ on olanzapine) suggested that the patient group may not have been truly 'treatment resistant'. '.

There are even more limited data on the efficacy of the newer SGA.s in treatment resistant schizophrenia. A study comparing quetiapine (600mg/day) and haloperidol (20mg/ day) in a population who had responded poorly or only partially to treatment with fluphenazine, showed a better response (20\% reduction in PANSS total score) at 8 weeks in the quetiapine group ( $52 \%$ compared to $38 \%$ ); these patients also experienced fewer side-effects (extra-pyramidal symptoms and need for anti-cholinergic medication). ${ }^{18}$ Aripiprazole is another new SGA with novel mechanism of action - it is a partial agonist at dopamine D2 receptors. There is no clear data on its effect in treatment resistance although one study suggested possible benefit in improving symptoms and quality of life. ${ }^{19}$

Determining any specific efficacy of SGAs other than clozapine on negative symptoms also needs to be examined; notably how much improvement is actually secondary to reduction in extra-pyramidal side-effects or improvement in mood, for example. Some studies of olanzapine, risperidone and amisulpride have "employed path analysis - a statistical technique that factors out the causes of secondary negative symptoms, including EPSE, depression and positive symptoms."'13 A review of several studies concluded that there was a suggestion that these SGAs have a beneficial effect on primary negative symptoms but that the evidence is not unequivocal. It highlighted that there are very few studies examining treatment response where negative symptoms predominate (also referred to as the deficit syndrome). ${ }^{13}$

The other group of studies that need to be considered are comparisons of atypicals with other atypicals. There are surprisingly few such studies, and again most have been of short duration. Moreover, they tend to have high response rates which suggest that the definition of treatment resistance may be too lenient. For example, in one study, patients had not been prospectively treated with a FGA to screen out treatment responders, and included patients who experienced side effects or ceased medication for reasons other than failed response. ${ }^{10}$

Bondolfi et al ${ }^{20}$ compared clozapine and risperidone in 86 treatment resistant patients over 8 weeks and showed similar response rates; Wahlbeck et al conducted a 10-week openlabel trial of risperidone compared with clozapine and showed no significant difference in response (20\% reduction in PANSS). ${ }^{21}$ Methodological limitations in both these studies have been outline in a recent review by Kerwin and Bolonna ${ }^{22}$ : respectively, that a rapid titration of clozapine may have led to intolerance and consequently suboptimal dose and that a very high drop-out of clozapine patients significantly reduced the power to detect a significant difference. Beuzen et $\mathrm{al}^{23}$ compared olanzapine and clozapine in 180 patients over 18 weeks, also showing similar efficacy between the two agents in terms of positive psychotic symptoms. A further study suggested that in another domain of treatment resistance, cognitive deficits, risperidone and olanzapine may be more effective than clozapine. ${ }^{24}$ However, this finding needs to be considered in light of the fact that different agents seem to have efficacy for different aspects of cognitive function. For example, clozapine may improve attention and verbal fluency but its benefit for working memory is less clear. ${ }^{25}$

\section{Augmentation Strategies}

The frequency of augmentation strategies used in treatment resistant schizophrenia in clinical practice does not reflect the scientific evidence that is available, as there are still few published randomised- controlled studies assessing such approaches. Although early studies suggested certain benefits for a variety of augmentation strategies, many of these have not been replicated. Goff et $\mathrm{al}^{5}$ suggest that this may reflect early publication bias (only positive results being published), false positive results due to multiple outcome measures in small patient samples, and differences in patient samples in terms of severity and stage of illness, as well as in past medication exposure. Most of the studies have been open label and uncontrolled. It is likely that augmentation may benefit some patient sub-groups rather than the treatment resistant population as a whole. Again, this emphasises the importance of clearly defining symptom domains in outcome trials and in clinical practice.

Augmenting one antipsychotic with another

As with most augmentation strategies there is a 'very limited research database' on combination antipsychotics. ${ }^{26}$ There is also the potential to increase the level of side-effects, with both agents contributing.

A recent review by Lerner et $\mathrm{al}^{27}$ of combination antipsychotic strategies in schizophrenia found 29 case- 
reports and case series (172 patients) and only one doubleblinded randomised placebo-controlled trial of 28 patients. These studies used a range of combinations including clozapine with risperidone, amisulpride, olanzapine or quetiapine; olanzapine with amisulpride or quetiapine; and risperidone with olanzapine or quetiapine. The authors concluded that some combinations may be effective but clearly further trials are required.

There have been case-reports of benefit from adding amisulpride to clozapine ${ }^{28}$, but we are aware of only one published double-blinded randomized controlled study examining this strategy in treatment resistant patients. Sulpride (a related compound) augmentation resulted in a significantly higher response (50\% - 8 of 16 patients) than placebo ( $8 \%$ - 1 of 12 patients) over 10 weeks. ${ }^{29}$ Here, response was defined as a $20 \%$ reduction in BPRS and included positive and negative symptoms. Thus cognition, disability, aggression or other domains were not reported specifically.

Risperidone is a relatively tenacious D2 blocker which should theoretically complement the relatively low D2 receptor affinity of clozapine. Thus, combining the two agents may reduce positive psychotic symptoms or at least enable a lower dose of clozapine and hence a reduced side-effect burden. There have been several case reports and uncontrolled trials of this strategy which have had mixed results. A double-blind, placebo controlled study by Josiassen et $a l^{30}$ compared clozapine/placebo to clozapine/risperidone (6mg) over a 12 week period in patients who had been partially responsive to clozapine. Both groups improved in terms of mean BPRS total and positive symptom subscale but that there was a significantly greater improvement in the clozapine/risperidone group. Interestingly there was also a significantly greater reduction in SANS score in the clozapine/ risperidone group. This was not replicated in another recent randomized, placebo-controlled, double-blind trial of augmentation of clozapine (>400mg for at least 12 weeks) with risperidone (3mg). With 'response' categorized as greater than $20 \%$ decline in total PANSS score, there was a $26 \%$ rate of response in the placebo arm compared with $18 \%$ response in the risperidone group. ${ }^{31}$

\section{Mood Stabilisers}

The addition of mood stabilizers such as lithium or sodium valproate to an antipsychotic is common in patients with a strong affective component to their illness or in those with a diagnosis of 'schizoaffective disorder' ${ }^{32}$ Of course these agents can also have their own side-effects - for example lithium can increase neurotoxicity, carbamazepine should not be prescribed with clozapine due to its potential for bone marrow suppression, and sodium valproate can further increase weight. While some studies suggest benefits from the addition of mood stabilisers to antipsychotics in patients with violent behaviour, and there are theoretical mechanisms such as these agents having an 'anti-kindling action' ${ }^{33}$, there is insufficient evidence to draw any definitive conclusions.

A Cochrane review of sodium valproate augmentation of antipsychotics in schizophrenia in 2004 concluded that, "there is some evidence for a more rapid improvement with valproate augmentation, but the effect vanished over time. ${ }^{34}$ Given this limited evidence, further large, well-designed trials are necessary. These might focus on people with schizophrenia with violent episodes, on those with treatment resistant symptoms and schizoaffective disorders." ${ }^{34}$ Lithium is traditionally cited as the best 'augmenter' but there have only been three positive small placebo controlled trials; ${ }^{5}$ subsequent studies have not replicated these outcomes. ${ }^{5}$ Furthermore, there are no well controlled studies combining lithium with atypical antipsychotics. In a review of carbamazepine augmentation in 2002 , the authors concluded that 'at present, this augmentation strategy cannot be recommended for routine use'. ${ }^{35}$

Lamotrigine, a glutamate antagonist, is an anti-convulsant which has efficacy in the treatment of depression in bipolar affective disorder. The theoretical basis of its use in treatment resistant schizophrenia is that excessive glutamate neurotransmission may contribute to positive symptoms. A recent double-blind, placebo-controlled trial over 14 weeks with a cross-over design, assessed the addition of lamotrigine to ongoing clozapine treatment in 34 treatment resistant patients. Lamotrigine was shown to be more effective than placebo in reducing positive symptoms and 'general psychopathological symptoms' measured by the PANSS, but had no significant benefit on negative symptoms. ${ }^{36}$

\section{Other Augmenting Agents}

Other augmentation strategies focus on specific 'target symptoms'. There have been several trials combining antidepressants and anti-psychotics and examining their impact on negative symptoms. These have included predominantly FGAs combined with SSRIs. They have shown some improvement in negative symptoms when fluvoxamine and fluoxetine have been used but there have been no consistent improvement with other SSRIs. ${ }^{5}$ A study by Berk and coworkers compared mirtazapine with placebo in addition to haloperidol in patients with stable schizophrenia but prominent negative symptoms; they showed a significantly greater reduction (43\%) in negative symptoms in the mirtazapine group with no significant effect on depressive symptoms. $^{37}$

Few studies have systematically examined the efficacy of antidepressant augmentation of SGA.s. One study ${ }^{38}$ combining fluoxetine and clozapine showed no benefit over clozapine alone. Furthermore, benefit in some cases may simply reflect increased antipsychotic levels via the antidepressant's inhibition of certain P450 isoenzymes. Certain combinations are particularly problematic in this regard; for example, fluvoxamine can cause a substantial increase in serum clozapine levels.

One more recent and novel augmentation strategy uses glutamatergic agents such as glycine and D-cycloserine. The rationale for such a strategy lies in the theory that one underlying mechanism in schizophrenia is a decrease in NMDA (glutamate receptor) activity. Glycine is a full NMDA receptor agonist and D-cycloserine is a partial agonist. Results with these agents as augmenting agents in patients with schizophrenia have thus far been mixed. Furthermore, pure NMDA agonists are potentially neurotoxic (due to Ca++influx); and co-agonists such as glycine require high doses to cross the blood-brain-barrier. ${ }^{5}$ One recent multi-centre study by Carpenter et $\mathrm{al}^{39}$ examined the efficacy of glycine and $\mathrm{d}$ cycloserine for the treatment of negative symptoms. It was a 
16-week randomized control trial of 171 patients receiving glycine, d-cycloserine or placebo in addition to their usual antipsychotic medication. There was a small improvement in both nonplacebo groups but there was no significant difference in the proportion of patients in each group who had a greater than 20\% change in symptom score (the chosen outcome measure). The authors concluded that the results did not support the efficacy or effectiveness of glycine or dcycloserine in the treatment of negative symptoms. Other augmentation strategies are summarised in Table III.

Thus, there is an emerging literature about a range of augmentation strategies which may be of benefit for a select set of symptom domains in certain patients. However, the benefit of most of these is still to be confirmed in well designed and sufficiently large trials. The potential additional side-effect burden associated with such strategies requires a careful risk-benefit analysis for each individual patient.

\section{Psychosocial Strategies}

Despite the promise of the newer antipsychotic medications and benefits that can accrue with targeted augmentation strategies (Table III), it is well recognised that many residual symptoms and disabilities can persist, even with optimal pharmacological treatment. Furthermore, given the broader concept of treatment resistance articulated above, we need to consider the range of other (non-pharmacological) treatments that have emerged over the past twenty years as part of the growth in rehabilitation psychiatry (Table IV).

\section{Intensive Case Management \\ Psychoeducation \\ Family Therapy \\ Cognitive Behavioural Therapy \\ "Compliance Therapy" \\ Social Skills Training \\ Cognitive Remediation}

Table IV: Examples of psychosocial treatments that seem to work

Arguably, these therapeutic strategies have particular relevance for negative and cognitive symptoms which often affect people pre-morbidly (prior to overt psychotic symptoms) and continue to impact on outcome in many ways - for example social isolation, poor adherence to medication and poor engagement with treatment services. Indeed, a review of twenty-four psychosocial intervention studies for schizophrenia found a greater beneficial effect of such therapies on negative symptoms than on positive symptoms. ${ }^{40}$

Studies of family therapy have consistently shown that it can lead to improved outcome in schizophrenia. The early impetus of these studies arose from the work of Brown and Rutter ${ }^{41}$ which examined the role of expressed emotion (EE) as a contributor to relapse in schizophrenia. Reducing expressed emotion within a family or other environment reduced relapse rates. More recent work suggests that intensive work specifically addressing expressed emotion may not be critical. Instead, basic psycho-education, family support, teaching the recognition of early warning signs and addressing issues of medication adherence may be equally effective and have an enduring effect. ${ }^{42}$ Again, most of the outcome measures in these studies have emphasised relapse and residual positive symptoms rather than other domains that may be equally relevant in treatment resistance.

Studies examining the role of social skills training tend to emphasise functional domains in addition to simply symptoms or relapse rates. Social skills training has been divided into basic personal skills, social problem-solving and cognitive remediation. ${ }^{42}$ Results of studies examining these different approaches have been mixed. Many studies show that basic skills can be taught and sustained with regular 'refresher sessions' but there is less clear evidence supporting generalisation of these skills to overall social functioning or integration. ${ }^{43}$

Other treatments such as cognitive behavioural therapy (CBT) have been shown to be effective at reducing residual positive symptoms. A review by Garety et $\mathrm{al}^{44}$ reported four controlled trials that showed that CBT decreased residual positive symptoms with some evidence of sustained benefit. There were reductions in relapse rates and enhanced engagement with other components of management plans. The authors concluded that further research is required to better understand interactions between different psychosocial interventions and between psychosocial treatments and medication. Others have emphasised the importance of "maximizing the synergy between pharmacotherapy and psychosocial therapies". ${ }^{42}$ 
'Compliance therapy' uses the principles of motivational interviewing and cognitive-behavioural approaches to psychosis in addition to addressing other relevant issues such as insight and stigma. In one of the first RCTs examining this issue, Kemp and colleagues compared treatment adherence, attitudes to treatment, insight and social functioning in a group of 74 patients with psychotic disorders who received either 'compliance therapy' or non specific counselling. ${ }^{45}$ The treatment group showed improvement in all these domains compared to the control group and the benefits were sustained over a 18-month follow-up period. It should be noted that the target group in this study was people who had recovered from an acute psychotic episode, so further research is required to specifically examine the efficacy of this intervention in a treatment resistant population.

Cognitive remediation therapy is one of several psychological approaches dealing with cognitive dysfunction in schizophrenia. Unlike some other, less effective methods which focus on directly teaching specific tasks, the emphasis of cognitive remediation is on 'providing strategies for dealing with problems in general'. ${ }^{46}$

Intensive/ assertive case management has been reviewed by Meuser et al..$^{47}$ Most consistent effects were for reduced hospital admissions and time in hospital and more stable accommodation. There was some improvement in symptoms and quality of life and little or unclear benefit on social or vocational functioning. Such results emphasise the importance of integrating such case-management models with vocational services. ${ }^{48}$

\section{Conclusions}

It is now well recognised that clinicians dealing with people with schizophrenia must appreciate the heterogeneity of the disorder and see people as individuals with their own strengths and vulnerabilities, in their particular family and broader social contexts. Such recognition has arisen from a range of factors such as de-institutionalisation and community care and the rise of the psychiatric consumer movement. It is probable that the heterogeneous nature of the illness has not been fully appreciated in much of the research in treatment resistant schizophrenia. Thus, it is plausible that some 'subgroups' may actually respond to a particular intervention but this has not been evident in research which may have included a broad range of illness types. There is a need for more research identifying predictors of 'responders' and 'nonresponders' to a particular intervention.

In this context, there has been considerable growth in research in a wider range of interventions, in particular in the psychosocial domain. A greater recognition of the multifaceted nature of treatment resistant schizophrenia and 'outcome' has been a significant advance in this area. However, there are still many limitations to our understanding of some of even the most elementary issues. There are even further challenges in more complicated areas such as consideration of the interactions of specific medications and other domains of management.

Thus, more holistic approaches have been adopted. An example is collaborative therapy (CLT) which is "a comprehensive framework for consumers, clinicians, services and others that can work systematically towards the achievement of optimal mental health outcomes". ${ }^{49}$ This approach is a multifaceted one, incorporating many of the treatments or treatment elements that have been shown to be effective in clinical trails (see above), but integrating them into a coherent longitudinal treatment framework. CLT has been adopted as a service delivery model in a number of sites in Australia, with encouraging outcomes. Further evaluation of such strategies is required.

\section{References}

1. Lindenmayer JP. Treatment refractory schizophrenia. Psychiatric Quarterly 2000;71(4):373-384.

2. Pantelis C, Lambert T. Managing patients with "treatmentresistant'schizophrenia. Med J Aust 2003;178(suppl May 5):s62-s66.

3. Liberman RP, Kopelowicz A. Recovery from schizophrenia: a challenge for the 21.st century. Int Rev Psychiatry 2002;14:245-255.

4. Peuskens J. The evolving definition of treatment resistance. J Clin Psychiatry 1999;60 Suppl 12:4-8

5. Goff DC, Freudenreich O, Evins AE. Augmentation strategies in the treatment of schizophrenia. CNS Spectrums 2001;6(11):904-911.

6. Fenton WS, McGlashan TH. Natural history of schizophrenia subtypes: positive and negative symptoms and long term course. Arch Gen Psychiatry 1991;48:978-86.

7. Andrews G, Sanderson K, Corry J, Issakidis C, Lapsley H. Costeffectiveness of current and optimal treatment for schizophrenia. Br J Psychiatry 2003;183:427-435.

8. Kane J, Honigfeld $G$, Singer J, Meltzer H. Clozapine for the treatment-resistant schizophrenic. A double-blind comparison with chlorpromazine. Arch Gen Psychiatry 1988;45:789-796.

9. Breier A, Hamilton SH. Comparative efficacy of olanzapine and haloperidol for patients with treatment-resistant schizophrenia. Biol Psychiatry 1999;45:403-411.

10. Chakos M, Lieberman J, Hoffman E, Bradford D, Sheitman B. Effectiveness of second-generation antipsychotics in patients with treatment-resistant schizophrenia: a review and meta-analysis of randomised trials. Am J Psychiatry 2001;158:518-526.

11. Therapeutic Guidelines: Psychotropic, Version 5, 2003. Therapeutic Guidelines Limited, Australia.

12. Schulte $P$. What is an adequate trial with clozapine?: therapeutic drug monitoring and time to response in treatment-refractory schizophrenia. Clin Pharmacokinet 2003;42(7):607-18.

13. Copolov DL, Castle D: Management of negative symptoms. In: Castle D, Copolov D, Wykes T, eds. Pharmacological and Psychosocial Treatments of Schizophrenia. London: Martin Dunitz Ltd, 35-49, 2003.

14. Kumra S, Frazier JA, Jacobsen LK, McKenna K, Gordon CT. Childhood-onset schizophrenia: a double-blind clozapinehaloperidol comparison. Arch Gen Psychiatry 1996;53:1090-1097.

15. Meltzer HY, Alphs L, Green AI, Altamura AC, et al. Clozapine treatment for suicidality in schizophrenia: International suicide prevention trial. Arch Gen Psychiatry 2003;60:82-92.

16. Wirshing DA, Marshall BD, Green MF, Mintz J, Marder SR, Wirshing WC. Risperidone in treatment-refractory schizophrenia. Am J Psychiatry 1999;1 56:1 374-1 379. Cited in Lindenmayer J-P. Treatment refractory schizophrenia. Psychiatric Quarterly 2000;71 (4):373-384.

17. Conley RR, Tamminga CA, Bartko JJ, Richardson C, Peszke M, Lingle J, Hegerty J, Love R, Gounaris C, Zaremba S. Olanzapine compared with chlorpromazine in treatment-resistant schizophrenia. Am J Psychiatry 1998;155:914-920.

18. Emsley R. Partial response to antipsychotic treatment: the patient with enduring symptoms. J Clin Psychiatry 1999;60(suppl 23):10-13. Cited in Lindenmayer J-P, Treatment refractory schizophrenia. 
Psychiatric Quarterly 2000;71(4):373-384.

19. Kane JM, Carson W, Kujawa M, Stringfellow J, Marcus R, Sanchez R, et al. Aripiprazole vs perphenazine in treatment-resistant schizophrenia (poster). Presented at the 156th American Psychiatric Association Meeting; May 17-22, 2003, San Francisco, California.

20. Bondolfi G, Dufour H, Patris M, May JP, Billeter U, Eap CB, Baumann P (Risperidone Study Group): Risperidone versus clozapine in treatment resistant chronic schizophrenia: a randomized doubleblind study. Am J Psychiatry 1998; 155:499-504.

21. Wahlbeck, $K$, Cheine, $M$, Tuiske, K. Risperidone versus clozapine in treatment-resistant schizophrenia: a randomized pilot study. Prog Neuropsychopharmacol Biol Psychiatry, 24, 911-922. Cited in Kerwin RW, Bolonna A. Management of clozapine-resistant schizophrenia. Adv in Psych Treatment 2005;11:101-106.

22. Kerwin RW, Bolonna A. Management of clozapine-resistant schizophrenia. Adv in Psych Treatment 2005;11:101-106.

23. Beuzen J-N, Birkett MA, Kiesler GM et al. Olanzapine vs clozapine: an international double-blind study in the treatment of resistant schizophrenia. 37th ACNP Annual Meeting, December 14-18, 1998. Cited in Lindenmayer J-P. Treatment refractory schizophrenia. Psychiatric Quarterly 2000;71(4):373-384.

24. Bilder RM, Goldman RS, Volavka J, Czobor P, Hoptman M, Sheitman B, Lindenmeyer JP, Citrome L, MCEvoy J, Kunz M, Chakos M, Cooper T, Horowitz TL, Lieberman JA. Neurocognitive effects of clozapine, olanzapine, risperidone, and haloperidol in patients with chronic schizophrenia or schizoaffective disorder. Am J Psychiatry 2002;1018-1028.

25. Wykes T, Castle D: Cognitive dysfunction in schizophrenia. In Castle D, Copolov D, Wykes T, eds. Pharmacological and Psychosocial Treatments in Schizophrenia. London: Martin Dunitz Ltd, 49-63, 2003

26. Castle D, Alderton D: Psychopharmacological management of schizophrenia. In: Castle D, Copolov D, Wykes T, eds. Pharmacological and Psychosocial Treatments in Schizophrenia. London: Martin Dunitz Ltd, 1-23, 2003.

27. Lerner V, Libov I, Kotler M, Strous RD. Combination of "atypical" antipsychotic medication in the management of treatment-resistant schizophrenia and schizoaffective disorder. Prog Neuropsychopharmacol Biol Psychiatry 2004;28(1):89-98.

28. Zink M, Knopf U, Henn FA, Thome J. Combination of clozapine and amisulpride in treatment-resistant schizophrenia - case reports and review of the literature. Pharmacopsychiatry 2004;37(1):26-31.

29. Shiloh R, Zeminshlany Z, Aizenberg D, Radwan M, Schwartz B, Dorfman-Etrog P, Modai I, Khaikin M, Weizman A. Sulpride augmentation in people with schizophrenia partially responsive to clozapine: a double-blind, placebo-controlled study. Br J Psychiatry 1997; 171:569-73.

30. Josiassen RC, Joseph A, Kohegyi E, et al. Clozapine augmented with risperidone in the treatment of schizophrenia: a randomized, double-blind, placebo-controlled trial. Am J Psychiatry 2005; 162:130-6.

31. Honer W, MacEwan GW, Williams R, Falkai P, McKenna PJ, PomarolClotet E, Chen EY, Leung SP, Wong J, Stip E. A randomized, placebocontrolled, double-blind trial of augmentation of clozapine with risperidone. International Congress on Schizophrenia Research 2005 (Abstract). Schizophr Bulletin 2005;31 (2):487.

32. Barnes TRE, MCEvedy CJB, Nelson HE. The management of treatment resistant schizophrenia unresponsive to clozapine. Br J Psychiatry 169(suppl 31):31-40.

33. Hosak L, Libiger J. Antiepileptic drugs in schizophrenia: a review. Eur Psychiatry 2002;17(7):371-8.

34. Basan A, Leucht S. Valproate for schizophrenia. Cochrane Database Syst Rev 2004;1.

35. Leucht S, McGrath J, White P, Kissling W. Carbemazepine augmentation for schizophrenia: how good is the evidence? J Clin Psychiatry 2002;63(3):218-24.

36. Tiihonen J, Hallikainen T, Ryynanen OP, Repo-Tiihonen E, Kotilainen I, Eronen M, Toivonen P, Wahlbeck K, Putkonen A. Lamotrigine in treatment-resistant schizophrenia: a randomized placebo-controlled crossover trial. Biol Psychiatry 2003;54:1241-1248.

37. Berk M, Ichim C, Brook S. Efficacy of mirtazapine add on therapy to haloperidol in the treatment of the negative symptoms of schizophrenia: a double-blind randomized placebo-controlled study. Int Clin Psychopharmacol 2001;16:87-92.

38. Buchanan RW, Kirkpatrick B, Bryant N, Ball P, Breier A. Fluoxetine augmentation of clozapine treatment in patients with schizophrenia. Am J Psychiatry 1996;1 153:1625-27. Cited in Goff DC, Freudenreich $O$, Evins AE. Augmentation strategies in the treatment of schizophrenia. CNS Spectrums 2001;6(11):904-911.

39. Carpenter WT, Buchanan RW, Javitt DC, Marder SR, Schooler NR, Heresco-Levy U, Kirkpatrick B, McMahon RP. Testing two efficacy hypothesis for the treatment of negative symptoms. International Congress on Schizophrenia Research 2005 (Abstract). Schizophr Bulletin 2005; 31 (2):478.

40. Mojtabai R, Nicholson Ra, Carpenter BN. Role of psychosocial treatments in management of schizophrenia: a meta-analytic review of controlled outcome studies. Schizophr Bulletin 24: 569-87.

41. Brown GW, Rutter M. The measurement of family activities and relationships: a methodological study. Hum Relations 1966;2(suppl):10-15.

42. Lauriello J, Lenroot R, Bustillo J. Maximising the synergy between pharmacotherapy and psychosocial therapies for schizophrenia. Psychiatr Clin N Am 2003;26:191-211.

43. Penn DL, Mueser KT. Research update on the psychosocial treatment of schizophrenia. Am J Psychiatry 1996;1 53:607-617.

44. Garety PA, Fowler D, Kuipers E. Cognitive-behavioural therapy for medication-resistant symptoms. Schizophr Bulletin 2000;26(1):7386.

45. Kemp R, Hayward P, Applewhaite G, Everitt B, David A. Compliance therapy in psychotic patients: a randomized control trial. $B M J$ 1996;312:345-9.

46. Wykes T, Castle D: Cognitive dysfunction in schizophrenia. In: Castle D, Copolov D, Wykes T, eds. Pharmacological and Psychosocial Treatments in Schizophrenia. London: Martin Dunitz Ltd, 49-63, 2003.

47. Meuser KT, Bond GR, Drake RE, Resnick SG. Models of community care for severe mental illness: a review of research on casemanagement. Schizophr Bulletin 1998:24(1):37-74.

48. Bell MD: Work and recovery in schizophrenia. In: Castle D, Copolov D, Wykes T, eds. Pharmacological and Psychosocial Treatments in Schizophrenia. London: Martin Dunitz Ltd, 11 9-120,2003.

49. Gilbert M, Miller K, Berk L, Ho V, Castle D. Scope for psychosocial treatments in psychosis: an overview of collaborative therapy. Australas Psychiatry 2003;11:113-26. 